\title{
What makes an author
}

\author{
Constructing a fair and accurate author list can be one of the most fraught aspects of manuscript publication. \\ We provide some advice and resources for authors at all career levels.
}

T: he acknowledgement of scientific contributions in the form of manuscript authorship is vital at all stages of a researcher's career, from the well-established principal investigator applying for million-dollar grants to the undergraduate student applying to $\mathrm{PhD}$ programs. It's essential that authorship lists are constructed with utmost care.

The variety of authorship practices across the scientific literature, however, is vast. Different fields, different countries, even different labs have different norms. Some practices are troubling: lab technicians not included for their major contributions to a study because they are not on an academic track; contributors removed from author lists due to personal disputes; researchers who have not substantially contributed added to papers (in a misguided attempt to increase 'impact') without their consent; senior scientists taking advantage of power imbalance to undeservedly gain publications.

Even researchers with the best intentions can struggle with finalizing a fair and accurate author list. Here, we provide some best practice guidelines and explain how Nature Methods handles authorship issues.

First of all, community guidelines for authorship are available. Nature Portfolio's authorship policies are based on guidelines developed by McNutt et al. (Proc. Natl Acad. Sci. USA 115, 2557-2560, 2018). Other guidelines in common use include those from the International Committee of Medical Journal Editors. As defined by Nature Portfolio, an author listed on a paper should have made a substantial contribution to the design of the work, the collection or analysis of data, the creation of a software tool, or the writing of the paper. This policy is meant to be broad and flexible, leaving "substantial contribution" up for quite a bit of room for interpretation.

In our view, job title or rank should never exclude a potential author. The lab technician or core facility scientist who developed a custom experimental workflow for the study should be included as an author. The first-year rotation student who spent several weeks collecting data should be included as an author. The software engineer who made substantial developments to an existing algorithm to analyze the data should be included as an author.

That said, not just any kind of assistance justifies authorship. People who provided routine services or basic technical help, contributed resources (such as by giving plasmids), proofread the manuscript, or gave general advice but did not otherwise significantly contribute to the scientific content of the paper should be thanked in the Acknowledgements. If previously published datasets or software tools are utilized in a new study without further development from their generators, there is no need to name them as authors. Even the person who secured funding need not necessarily be an author on a paper-they too ought to have scientifically contributed in a meaningful way. This is almost unheard of in lab-based science, where a principal investigator typically supervises the design of experiments and analysis of the resulting data, but it's relatively common practice in, for example, computer science for grad students to publish sole-author papers.

Though different research fields have different traditions, the custom in life sciences research is to name the person or people who did the bulk of the research first, followed by other contributors in descending order of the significance of their contributions, with the principal investigator(s) named at the very end of the list. Disputes often arise over who is named first on a paper. Most journals allow co-first-authorship designations to recognize cases of equal contribution, but one name must necessarily come first; the research community should take care to recognize these equal contributions. Those listed second should not feel that their contributions are minimized in any way.

Project managers should make defining authorship and authorship order a priority of a new study. Students and postdocs, collaborators, and service providers should speak up if authorship is not discussed early on. Setting clear parameters and communicating openly from the outset of a research study-in some cases even by signing formal authorship agreements-can go a long way toward preventing disputes and hurt feelings down the line.

All authors on a paper have a responsibility for at least part of its content. Nature Portfolio journals require author contribution statements, which in our view are crucial to clarify each author's role and responsibilities, to assign credit where it is due, to discourage the practice of including authors who did not significantly contribute to the study, and to assign accountability in (rare) cases of misconduct. The corresponding author, the main point of contact with a journal, has extra responsibilities. They are tasked with communicating with all coauthors at the submission, revision and final acceptance stages, including ensuring that all are satisfied with the manuscript text and content. The corresponding author must also check that all coauthors agree with changes to the author list, that any competing interests are declared, and that the paper complies with all of the journal's policies regarding data, materials and code sharing. Note that the journal corresponding author need not be the same person as the corresponding author(s) listed on the published paper, who take responsibility for post-publication inquiries.

We encourage our authors to speak up to let us know when best practices for authorship are not being followed. However, our editorial power is limited to delaying review or publication until disputes can be resolved, making corrections to papers, adding an 'editorial expression of concern' or, in very rare cases, retracting a paper. We rely on authors to behave responsibly and we cannot investigate or adjudicate authorship disputes. We advise those embroiled in disputes to seek help from their department head, university or other employer. We also recommend speaking to an experienced neutral party familiar with the study for advice-it's human nature to often overestimate our own contributions, but it's right to speak up about unfair treatment.

Unfortunately, we do not have the space to cover all possible authorship scenarios in this short piece. We look forward to answering your questions and perhaps sparking some lively discussion on Twitter, where you can follow us at @naturemethods.

Published online: 3 September 2021 https://doi.org/10.1038/s41592-021-01271-8 